\title{
Manufactura de un prototipo cabezote de motor de dos tiempos mono cilíndrico 100 cc aplicando ingeniería inversa y el método CAD/CAM
}

\author{
Manufacture of a head prototype of a $100 \mathrm{cc}$ single cylinder two-stroke \\ engine applying reverse engineering and the CAD / CAM method.
}

Victor David Bravo Morocho. ${ }^{1}$, Edison Patricio Abarca Pérez. ${ }^{2}$, Santiago Alejandro López Ortíz. ${ }^{3} \&$ Jhon Jesus Freire Bravo. ${ }^{4}$

Recibido: 21-06-2021 / Revisado: 30-06-2021 /Aceptado: 18-07-2021/ Publicado: 05-08-2021

\begin{abstract}
.
DOI: https://doi.org/10.33262/concienciadigital.v4i3.1.1823

A head prototype of a 100cc single cylinder two-stroke engine was manufactured using reverse engineering and the CAD / CAM method, the research begins with the mechanical and chemical characterization of a reference head (AX100), to then geometrize it using Computer Aided Design (CAD) software and print it on a 3D printer, once the printed mold is obtained, the preparation proceeded of the sand with calcium silicate, where it was molded and carbon dioxide was injected to harden the mold, they were joined and the aluminum was melted, $1.92 \%$ zinc, $1.35 \%$ silicon and $1.62 \%$ were added. of copper in the casting, sodium and tin were also added to remove the gases and obtain a more fluid casting until reaching a temperature of 1000 degrees Celsius, finally, the cast head was machined on a 3-axis machining center and an on-site test was performed on a test internal combustion engine; a prototype head was obtained with a 392 series aluminum with an average hardness of $110 \mathrm{HB}$ fully functional and with characteristics similar to

1 Escuela Superior Politécnica de Chimborazo, Facultad de Mecánica, Riobamba, Ecuador, email victor.bravo@espoch.edu.ec, https://orcid.org/0000-0001-5629-259X

2 Escuela Superior Politécnica de Chimborazo, Facultad de Mecánica, Riobamba, Ecuador, email edison.abarca@espoch.edu.ec, https://orcid.org/0000-0001-7041-4805X

${ }^{3}$ Escuela Superior Politécnica de Chimborazo, Facultad de Mecánica, Riobamba, Ecuador, email sa_lopez @ espoch.edu.ec, https://orcid.org/0000-0002-6314-6299X

4 Escuela Superior Politécnica de Chimborazo, Facultad de Mecánica, Riobamba, Ecuador, email jhon.freire@espoch.edu.ec, https://orcid.org/0000-0002-1299-6285X
\end{abstract}


the reference head; it is recommended to use the green casting method since it allows obtaining complex geometries and considering the mass composition of each alloying element since when aluminum is melted these evaporate around 2 to $3 \%$.

Keywords: Aluminum alloy, head, automotive engineering, reverse engineering, CAD/CAM method.

\section{Resumen.}

Se manufacturo un prototipo de cabezote de un motor de dos tiempos mono cilindro 100cc aplicando ingeniería inversa y el método CAD/CAM, la investigación inicia con la caracterización mecánica y química de un cabezote de referencia (AX100), para seguidamente geometrizarlo mediante un software de Diseño Asistido por Computadora (CAD) e imprimirlo en una impresora 3D, una vez obtenido el molde impreso se procedió a la preparación de la arena con silicato de calcio, donde se moldeo y se procedió a inyectar dióxido de carbono para endurecer el molde, se unieron y se procedió a fundir el aluminio, se agregó $1.92 \%$ de zinc, $1.35 \%$ de silicio y $1,62 \%$ de cobre en la colada además se agregaron sodio y estaño para sustraer los gases y obtener una colada más fluida hasta alcanzar una temperatura de 1000 grados Centígrados, finalmente se mecanizo el cabezote fundido en un centro de mecanizado de 3 ejes y se realizó una prueba insitu en un motor de combustión interna de pruebas; se logró obtener un prototipo de cabezote con un aluminio de la serie 392 con una dureza promedio de 110HB totalmente funcional y con características similares al cabezote de referencia; se recomienda utilizar el método de fundición en verde ya que permite obtener geometrías complejas y considerar la composición másica de cada elemento aleante ya que al fundir el aluminio estos se evaporan alrededor de un 2 a 3\% (Freire Bravo, 2021).

Palabras claves: Aleaciones ligeras; aluminio; cabezote; ingeniería inversa; método $\mathrm{CAD} / \mathrm{CAM}$.

\section{Introducción.}

La producción manufacturera del ecuador ha sido de poco interés y de menor porcentaje en el campo automotriz debido a los repuestos importados a precios bajos y accesibles a los usuarios, principalmente las autopartes de recambio del motor de combustión interna (Freire Bravo, 2021). En el caso de las partes y piezas de recambio del tren propulsor de las motocicletas se cuenta así mismo con todos los repuestos del componente, pero para los casos de los motores de motos de dos tiempos se cuenta con pocos repuestos de los cuales, un repuesto del motor de moto de dos tiempos es el cabezote, este componente por su forma tiene mayores inconvenientes de manufactura y está muy bajo de repuestos. (Aeade, 2020).

La investigación se enfoca en el diseño y construcción de un cabezote de un motor de dos tiempos mono cilíndrico mediante la utilización de la ingeniería inversa y métodos 
CAD/CAM, ya que, en Ecuador no se cuenta con procesos de manufactura de piezas automotrices conformantes de un motor de combustión interna tanto móviles como fijas, por tal razón, se manufacturo un cabezote aplicando métodos de desagregación tecnológica automotriz (Freire Bravo, 2021).

Así, el presente proyecto permitirá mostrar los procesos que se requieren para obtener un cabezote con las mismas características de un producto importado de características similares, acogiéndose a las normas nacionales e internacionales que permitan caracterizar un cabezote, así como también, los procesos de manufactura para profundizar los conocimientos teóricos sobre los procesos de diseño CAD/CAM, así mismo en los procesos de especificación del material que se deberá utilizar para la obtención de la pieza e incentivar a la población local en la manufactura de piezas automotrices (Freire Bravo, 2021).

\section{Cabezote de motor de dos tiempos.}

El cabezote o culata dispone del sistema de refrigeración aleteado si es por aire, y con conductos de circulación de líquido en el caso de ser la refrigeración por líquido. Hay culatas refrigeradas por líquido que incorporan un culatín (Escudero S, 2011).

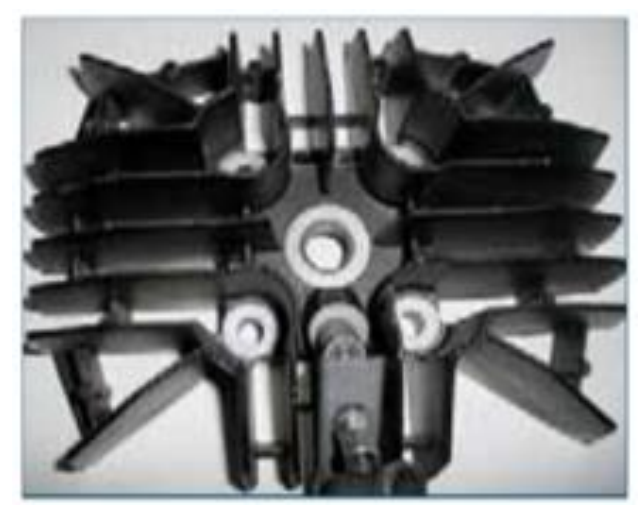

Figura 1. Composición de microestructura. Fuente: Escudero, S. 2011.

Fabricación de las culatas.

Las culatas de fundición de hierro se siguen empleando en grandes motores, como en maquinaria de obras públicas, camiones, etc., donde las culatas son de grandes dimensiones y, por ello, más propensas a alabeos. En estos casos se elige la fundición de hierro porque es más resistente que las aleaciones ligeras de aluminio (Freire Bravo, 2021).

Las aleaciones ligeras tienen la ventaja de reducir el peso y, sobre todo, de tener un alto coeficiente de disipación térmico. Sus principales inconvenientes son la menor resistencia al alabeo con el calor.

Las aleaciones ligeras llevan aleados aluminio, magnesio y silicio. Este último da resistencia a la culata, aunque dificulta los mecanizados posteriores. Estas culatas son, con diferencia, las más empleadas en la actualidad. Los procesos de fabricación de las 
aleaciones ligeras son las fundiciones en coquilla a baja presión y, las más modernas, de molde positivo o perdido, llamado también lost foam (Escudero S, 2011).

\section{Ingeniería inversa.}

Se interpreta a la ingeniería inversa como la técnica de copiado de geometrías en diferentes aplicaciones artísticas, prototipado de elementos mecánicos, etc. Sin embargo, la ingeniería inversa es el proceso mediante el cual, el diseñador parte de la existencia de un sistema, elemento, o aplicación de ingeniería en cuestión para realizar su reproducción e innovación de forma eficiente y rápida, en especial cuando se trata de geometrías complejas (Monroy M, 2013).

Entre las aplicaciones más importantes de la ingeniería inversa mediante la digitalización de objetos, es el diseño y prototipado para la fabricación de elementos mecánicos en el campo aeronáutico y automotriz en la copia y réplicas de modelos, en la generación de nuevos productos, en el mantenimiento e inspección dimensional en el desgaste de elementos mecánicos, en la manufactura y reconstrucción de prótesis médicas, aplicaciones electrónicas, etc. Sin embargo, como se mencionó anteriormente, la reproducción de geometrías no es suficiente, es necesario la utilización de tecnologías de manufactura, para lograr la reproducción y manufactura de los elementos mecánicos en la industria automotriz. Por lo tanto, la geometría adquirida es necesario que se lleve a un proceso de manufactura, mediante impresión 3D, o procesos de inyección y/o mecanizado para lograr construir los elementos mecánicos en estudio, para lo cual, se utiliza software de diseño que permiten procesar la geometría copiada mediante herramientas de manufactura computarizados como CAD, CAM y CAE, que son parte de los diferentes procesos de ingeniería directa e ingeniería inversa para lograr alcanzar resultados confiables (Villarreal C, 2019).

\section{Normas internacionales.}

Las normas son acuerdos documentados, generalmente voluntarios, en los que se establecen criterios para productos, servicios y procedimientos. Mediante las normas se garantiza que los productos y servicios sean adecuados, comparables y compatibles para la finalidad prevista (Gmbh P, 2018).

La norma UNE EN 12890:2001, establece las distintas calidades de los moldes en función de los materiales que estén constituidos. La madera es el material más común por su facilidad en la generación del modelo. El recubrimiento de su superficie con una fina capa de pintura facilita su extracción pues evita su adhesión con la arena en verde, confiriendo además impermeabilidad. El ángulo de salida para el desmoldeo para moldes de madera se encuentra entre $3^{\circ}$ y $0,5^{\circ}$. Así mismo se establecen las sobremedidas para el mecanizado de piezas fundidas tanto ferrosas como no ferrosa (García J, 2016). 


\section{Metodologia.}

Determinación de las propiedades mecánicas del cabezote de un motor mono cilindro de 100cc aplicando ingeniería inversa.

Para determinar las propiedades mecánicas del cabezote de referencia se realiza las 4 pruebas fundamentales para caracterizar el material, siendo el ensayo de metalografía, micrografía, espectrometría y dureza como se muestra a continuación (Freire Bravo, 2021).

ASTM E10-01 (Standard Test Method for Brinell Hardness of Metallic Materials), ASTM ASTM E3 -01 (Standard Practice for Preparation of Metallographic Specimens), ASTM E407 -07(2015) e1 (Standard Practice for Microetching Metals and Alloys), -ASTM E1251 -17a (Standard Test Method for Analysis of Aluminum and Aluminum Alloys by Spark Atomic Emission Spectrometry), •ASTM E350 -18 (Standard Test Methods for Chemical Analysis of Carbon Steel, Low-Alloy Steel, Silicon Electrical Steel, Ingot Iron, and Wrought Iron) (Bravo Morocho V.D, Abarca Pérez E.P, Escobar Guachambala M.A, 2020)

\section{Ensayo de metalografía y micrografía.}

Se obtuvo una probeta de una aleta del cabezote de referencia y se preparó según la norma ASTM E3-11, 2017.

Para realizar las pruebas se realizó el ataque químico con Nital a la probeta y se procedió a observar en el microscopio, donde se obtuvo la micrografía y análisis según la norma ASTM E112-13 como se puede ver en la Figura 2 a continuación.

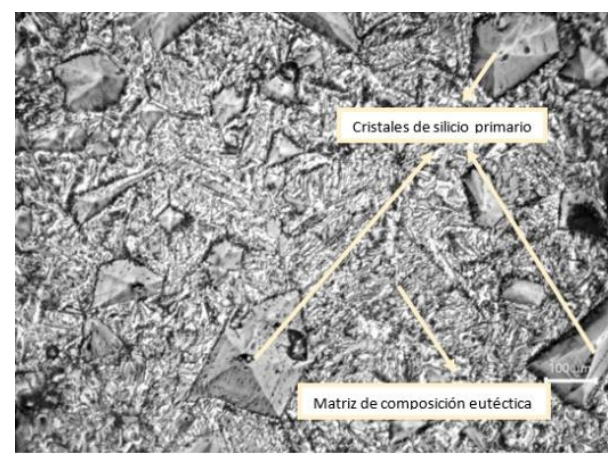

Figura 2. Composición de microestructura.

Fuente: Escudero, S. 2011.

Mediante la micrografía obtenida en la figura 2 por el método comparativo se observó y analizó que es una aleación de aluminio - silicio en la cual se tiene cristales de silicio con una matriz de composición eutéctica. Así mismo mediante la aplicación del software ImageJ se obtuvo los porcentajes de Silicio en el cual se estima en un 22,17\% de Silicio en la micrografía. Determinando que se trata de una aleación EZL18 (Wang R, 2012). 


\section{Ensayo de espectrometría.}

Una vez preparada la probeta se procede a realizar el estudio mediante la norma ASTM E716-16, en el espectrómetro BRUKER Q2 ION, donde se realizó el ensayo, dando como resultado la Figura 3 a continuación.

\begin{tabular}{|c|c|c|c|c|c|}
\hline \multicolumn{6}{|c|}{ Sample: } \\
\hline \multicolumn{2}{|c|}{ Analysis Time: } & \multirow{2}{*}{$\begin{array}{r}09.05 .201916: 42: 13 \\
\mathrm{Fe}[\%]\end{array}$} & \multicolumn{3}{|c|}{ Method: Al120 } \\
\hline & Si [\%] & & $\mathrm{Cu}[\%]$ & Mn [\%] & $\mathrm{Mg}[\%]$ \\
\hline \multirow[t]{2}{*}{$\varnothing$} & 18,32 & 1,054 & 2,093 & 0,184 & 0,072 \\
\hline & $\mathrm{Cr}[\%]$ & $\mathrm{Ni}[\%]$ & $\mathrm{Zn}[\%]$ & Ti [\%] & $\mathrm{Pb}[\%]$ \\
\hline \multirow[t]{2}{*}{$\varnothing$} & 0,014 & 0,025 & 0,218 & 0,046 & 0,186 \\
\hline & Sn [\%] & $\mathrm{V}[\%]$ & $\operatorname{Sr}[\%]$ & $\mathrm{Zr}[\%]$ & $\mathrm{Co}[\%]$ \\
\hline \multirow[t]{2}{*}{$\varnothing$} & 0,026 & 0,011 & $<0,0030$ & 0,0064 & $<0,0030$ \\
\hline & $\mathrm{Ca}[\%]$ & Al [\%] & & & \\
\hline$\varnothing$ & 0,020 & 77,72 & & & \\
\hline
\end{tabular}

Con los resultados obtenidos en el Gráfico 1, se procede a comparar con los porcentajes y se constata que en la tabla que expone (Millán F, 2016) se trata de una aleación de aluminio de la serie 3XX.X de moldeo (Freire Bravo, 2021).

\section{Ensayo de dureza.}

En el ensayo de dureza se utilizó la probeta que se realizó el análisis metalográfico y se analizó según la norma ASTM E10-18, 2018.

Se coloco la probeta en el durómetro con un identador de carburo de tungsteno de $2.5 \mathrm{~mm}$, se procedió a la prueba y con una carga de $613 \mathrm{~N}$ se obtiene una huella en la probeta, en la cual se observó en un microscopio y se obtuvieron las medidas longitudinales de la huella en la probeta 11 y 12 . Mediante la aplicación de la fórmula para obtener la dureza Brinell (1) se procede a reemplazar los datos para realizar su respectivo calculo (Freire Bravo, 2021).

Datos:

$P=613 \mathrm{~N}=\frac{613}{9.81}=62.487 \mathrm{kgf}$

$D=2.5 \mathrm{~mm}$

$d=\frac{0.82+0.87}{2}=0.845 \mathrm{~mm}$

$l 1=0.82 \mathrm{~mm}$

$l 2=0.87 \mathrm{~mm}$ 
Donde:

$P=$ Carga aplicada

$D=$ Diametro de la bola en $\mathrm{mm}$

$d=$ Promedio de huella $l 1$ y $l 2$

l1 = Medida de un lado de la huella

l2 = Medida de un lado de la huella

$H B=\frac{2 P}{\pi D^{2}}\left(\frac{1}{1-\sqrt{1-\frac{d^{2}}{D^{2}}}}\right)$

$H B=\frac{2 * 62.486 \mathrm{kgf}}{\pi(2.5 \mathrm{~mm})^{2}}\left(\frac{1}{1-\sqrt{1-\frac{(0.845 m m)^{2}}{(2.5 m m)^{2}}}}\right)$

$H B=108.389 \cong 110 H B$

Con la dureza Brinell de 110HB (2) se confirma que es un aluminio aleado y con los ensayos antes afirmando una aleación de la serie 392 según ASM H, 2004.

Geometrización y obtención de los moldes de fundición, códigos ISO y elaboración de planos.

En esta sección se realizan los procedimientos previos para poder realizar la fundición del cabezote ya que se creó un cabezote con las mismas dimensiones y con las mismas características tanto físicas como mecánicas (Freire Bravo, 2021).

Se empezó con el geometrizado de la parte inferior del cabezote siendo la parte esencial del elemento la cual se asientan las aletas de enfriamiento (Freire Bravo, 2021).

Una vez geometrizado la base se procedió a geometrizar las aletas y se realizaron los respectivos acabados tanto de inclinaciones como de redondeados (Freire Bravo, 2021).

Para el porceso de obtencion de moldes se tomo en cuenta la contracicon del material de fundicion por lo que en la tabla que expone (Rocabruna J, 2018) se realizara un incremento de las dimensiones del cabezote geometrizado de $3 \%$ y se modificara el cabezote mediante la sustracción de la aleta superior y de las tapas inferiores para facilitar la impresión 3D (Freire Bravo, 2021).

Así mismo se separaron la aleta superior y las tapas inferiores y se procedió a realizar el corte laser como se muestra en la Figura 3, a continuación. 


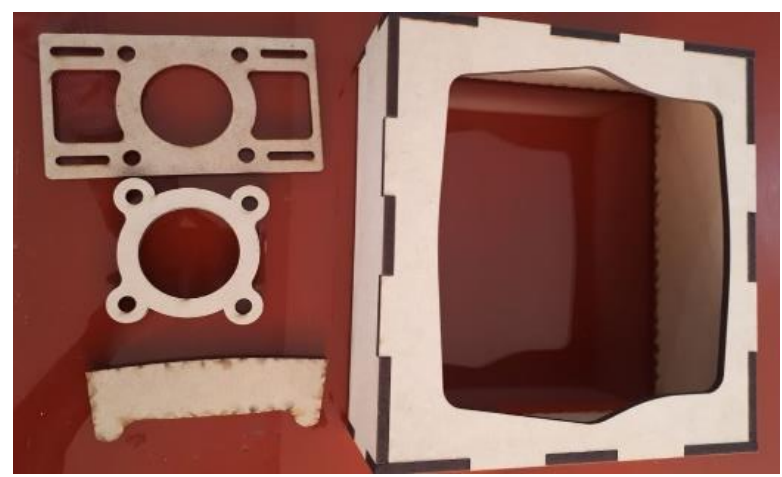

Figura 3. Tapas y caja realizadas en corte laser. Fuente: Elaboración propia.

Para la obtención de los códigos ISO se realizó en el software Siemens Nx11, en el cual se realizaron las operaciones para mecanizar la cámara de combustión, en la cual se empezó con el aplanado de la superficie, se perforo el orificio central para la bujía, se realizó una primera pasada en la cavidad, una vez terminado ese proceso se realiza una segunda pasada y para culminar la cámara de combustión se realiza un acabado, se termina el proceso de mecanizado con las perforaciones de los agujeros de los pernos que se sujetan al cabezote. Por lo que una vez realizadas estas operaciones se procedió a la verificación de las operaciones como se muestra en la Figura 4, verificando que no haya choques de las herramientas como en las trayectorias. Una vez realizado este proceso se obtuvo los códigos ISO (Freire Bravo, 2021).

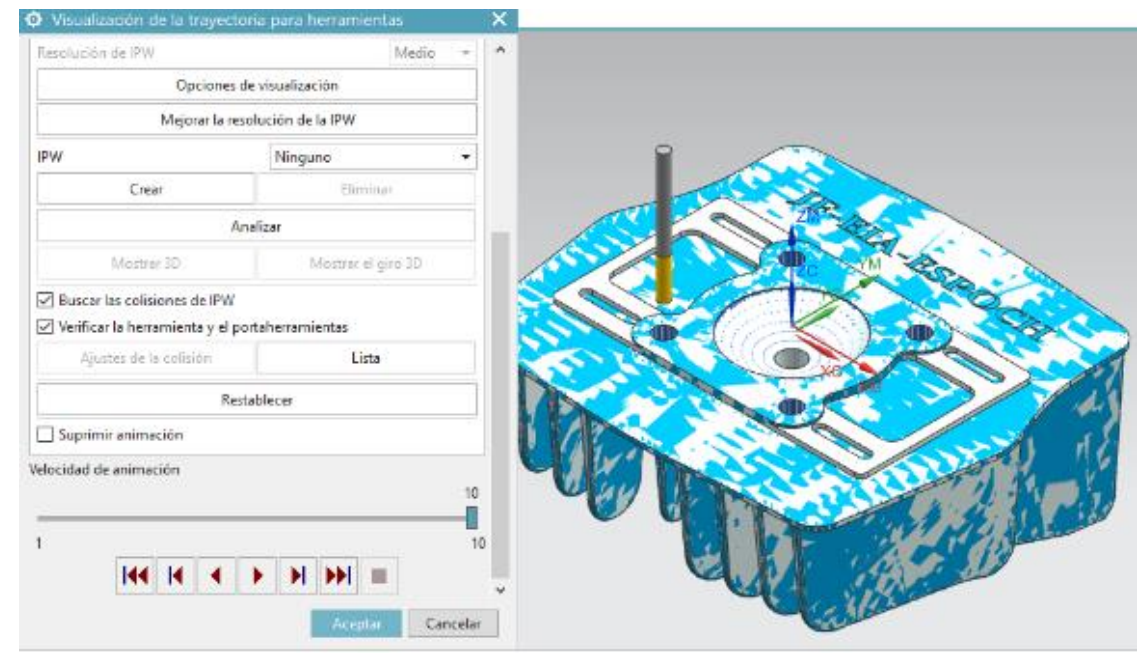

Figura 4. Perforado de cavidades de sujeción de pernos.

Fuente: Elaboración propia.

Con el prototipo de cabezote geometrizado se realizaron los planos constructivos de las vistas principales como se ve en la Figura 5, donde se detallan todas las medidas, ángulos de inclinación, redondeos y acabados superficiales que tiene el cabezote, así mismo todos los detalles del cabezote tanto interna como externamente (Freire Bravo, 2021). 

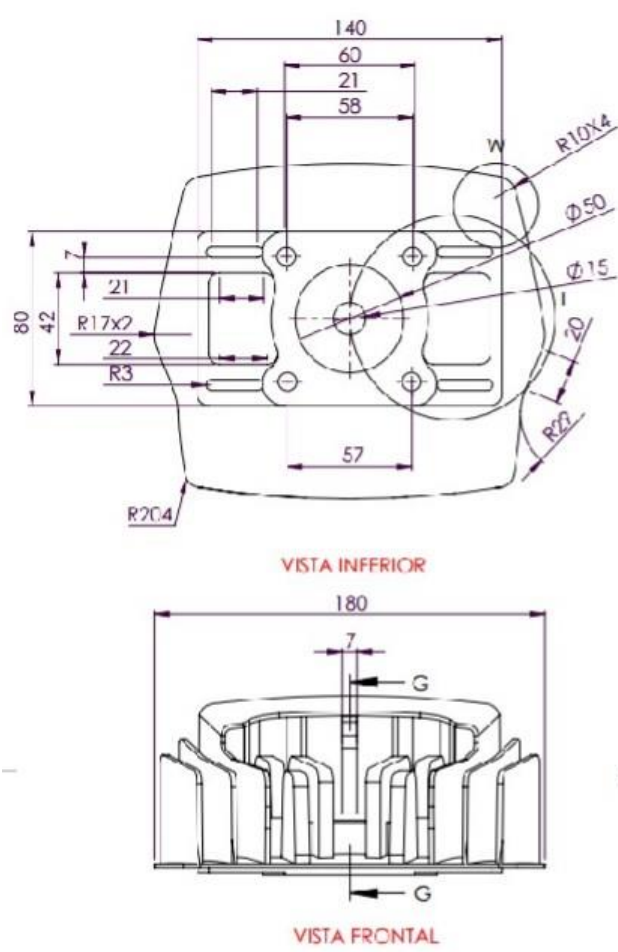

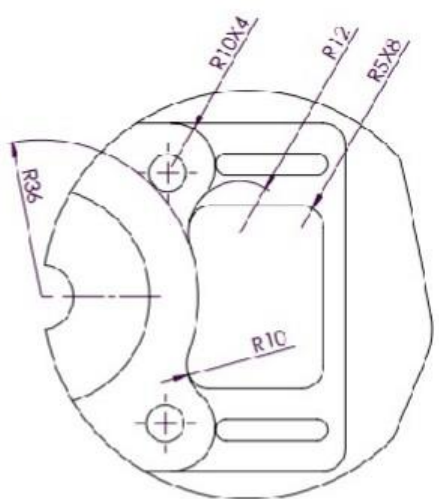

DETALLE!
ESCALA I :

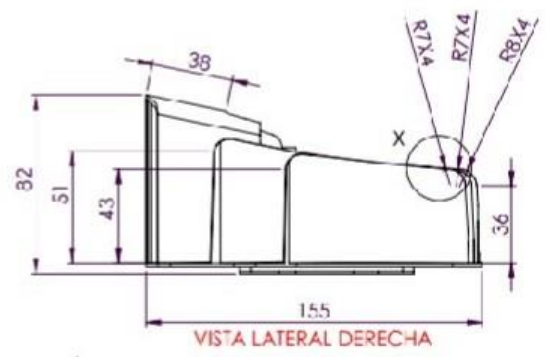

Figura 5. Planos constructivos del prototipo de cabezote. Fuente: Elaboración propia.

Manufactura del cabezote de motor empleando técnicas de fundición y maquinado CNC.

Se inicia con el moldeo en arena donde se realizan los respectivos cálculos de volumen de la mazarota (3), diámetro de la mazarota (4) y módulo de enfriamiento de la mazarota (5) (Freire Bravo, 2021).

Datos del cabezote geometrizado:

$V_{\text {pieza }}=358786.91 \mathrm{~mm}^{3}$

$A_{\text {pieza }}=201919.91 \mathrm{~mm}^{2}$

\section{Mazarota:}

Volumen mínimo:

$V_{\text {maz }}=k . c . V_{\text {pieza }}$

$V_{\text {maz }}=2 x\left(\frac{3.8}{100}\right) \times 358786.91 \mathrm{~mm}^{3}$

$V_{m a z}=27267.80 \mathrm{~mm}^{3}$

Diámetro de mazarota:

$D_{\text {maz }}=\sqrt[3]{\frac{2 V_{\operatorname{maz}}}{\pi}}$ 
$D_{\text {maz }}=\sqrt[3]{\frac{2 x\left(27267.80 \mathrm{~mm}^{3}\right)}{\pi}}$

$D_{\text {maz }}=25.89 \mathrm{~mm}$

Módulo de enfriamiento:

$M_{\text {maz }}=1.2 x M_{\text {pieza }}$

$M_{m a z}=1.2 x\left(\frac{358786.91 \mathrm{~mm}^{3}}{201919.91 \mathrm{~mm}^{3}}\right)$

$M_{m a z}=2.13 m m$

Donde:

$D_{\text {maz }}=$ Diametro de mazarota

$V_{\text {maz }}=$ Volumen de mazarota

$k=$ Factor de seguridad $=2$

$c=$ Coeficiente de contraccion del material

$V_{\text {pieza }}=$ Volumen de pieza

$M_{\text {maz }}=$ Modulo de mazarota

$M_{\text {pieza }}=$ Modulo de pieza

Dimensiones de la mazarota:

$D_{m a z}=26 m m$

$h_{m a z}=52 \mathrm{~mm}$

$D_{m a z}=27608.31 \mathrm{~mm}^{3}$

$A_{m a z}=5309.29 \mathrm{~mm}^{2}$

Para el cálculo del bebedero se toma en cuenta la ley de Torricelli (6), así mismo se calcula el diámetro base del bebedero tanto el diámetro 2 (7) como el diámetro 1 (8).

\section{Bebedero:}

Se asume una altura de salto del bebedero de $h_{2}=60 \mathrm{~mm}=H$.

$V_{2}=\sqrt{2 \cdot g \cdot H}$ 
$V_{2}=\sqrt{2\left(9.81 \mathrm{~m} / \mathrm{s}^{2}\right)\left(\frac{60 \mathrm{~mm}}{1000 \mathrm{~mm}}\right)}$

$V_{2}=1.085 \mathrm{~m} / \mathrm{s}$

\section{Diámetro base del bebedero:}

$D_{2}=\frac{R e . \eta}{V_{2} \cdot \rho}$

$D_{2}=\frac{10000 \times 0.003233 \mathrm{~kg} / \mathrm{ms}}{1.085 \mathrm{~m} / \mathrm{s} \times 2700 \mathrm{~kg} / \mathrm{m}^{3}}$

$D_{2}=0.011 \mathrm{~m}=11.03 \mathrm{~mm}=12 \mathrm{~mm}$

$D_{1}=D_{2} \sqrt[4]{\frac{h_{2}}{h_{1}}}=11.03 m m x \sqrt[4]{\frac{60 m m}{20 m m}}$

$D_{1}=14.52 \mathrm{~mm}=15 \mathrm{~mm}$

Donde:

$R e=$ Numero de Reynolds ideal $=10000$

$\eta=$ Viscosidad Dinamica $=0.003233 \mathrm{~kg} / \mathrm{ms}$

$\rho=$ Densidad Liquida $=2700 \mathrm{~kg} / \mathrm{m}^{3}$

\section{Dimensiones de bebedero:}

$D_{\text {beb.i }}=12 \mathrm{~mm}$

$D_{\text {beb.s }}=15 \mathrm{~mm}$

$h_{b e b}=60 \mathrm{~mm}$

$V_{b e b}=8623.67 \mathrm{~mm}^{3}$

$A_{b e b}=2835.30 \mathrm{~mm}^{2}$

Luego se procedió con la preparación del molde base en el cual se realizaron las inclinaciones estas se realizaron aproximadamente a $3^{\circ}$ de inclinación, además se colocó cera y se aplicó talco para evitar que la arena preparada se pegue en el molde. Después se procedió a preparar la arena para moldear la cual paso por una zaranda para quitar todos los elementos grandes y dejarle totalmente fina, y así mezclar con aglutinante en este caso silicato de calcio, hasta obtener una masa húmeda de arena. Luego de preparar la masa de arena se procedió a moldear el cabezote por lo cual se fue rellenando las cajas del molde de una en una y se aplicó $\mathrm{CO}_{2}$ para endurecer el molde de arena y evitar quebraduras. Una vez realizado los moldes se procede a quitar la humedad mediante un soplete (Freire Bravo, 2021). 
Una vez secados los moldes se procedió a calentar el hormo y a aplicar el aluminio tanto de cable de tendido eléctrico como material reciclado de cabezotes. Así mismo se empezó aplicar estaño para que el aluminio tenga mayor fluidez al momento de vaciarlo, también se agregó como fundente sodio para eliminar los gases que tiene el aluminio. Al constatar que la fundición alcanzo los $1000^{\circ} \mathrm{C}$ se procede a verter en los moldes ya preparados y se espera hasta que se enfríen a temperatura ambiente. Una vez enfriados los moldes fundidos se procede a separar los moldes y se obtiene una pieza fundida con excelentes acabados. Después de haber separado el molde de arena se procedió a quitar el material excedente del cabezote mediante una fresadora universal, en esta fresadora se aplano la superficie en la cual se procederá a mecanizar mediante lectura de códigos (Freire Bravo, 2021).

Al haber acabado de mecanizar se obtiene una superficie plana en la cual se referenciará el punto de partida para el mecanizado CNC. Así mismo se procedió a colocar el cabezote en una fresadora $\mathrm{CNC}$ de 3 ejes y se procedió a la lectura de los códigos, con las herramientas preseleccionadas para el trabajo de mecanizado (Freire Bravo, 2021).

\section{Resultados.}

Al haber concluido todos los procesos de obtención del cabezote se procedió a la comparación física y química de los cabezotes de referencia y prototipo, en el cual se obtuvo los siguientes resultados. Con respecto a la forma del cabezote se tiene un cabezote idéntico en forma y tamaño como se puede ver en la Figura 6, a continuación (Freire Bravo, 2021).

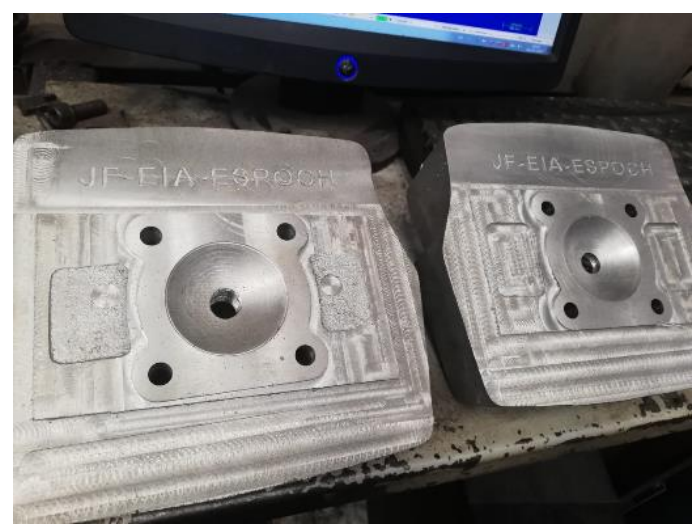

Figura 6. Comparación de cabezote de referencia con el prototipo.

Fuente: Elaboración propia.

Así mismo se realizaron las pruebas de espectrometría al cabezote prototipo como se muestra en el Gráfico 2, donde se obtuvo un aproximado de la aleación de referencia del cabezote de referencia como se ve en el Gráfico 3, en la cual debido a la oxidación y evaporación de los elementos químicos de estos aleantes, estos perdieron un porcentaje en la fundición, sin embargo, están dentro del rango permitido en cuanto a composición química de cabezotes (Freire Bravo, 2021). 


\begin{tabular}{|c|c|c|c|c|c|}
\hline \multicolumn{6}{|c|}{ Sample: } \\
\hline & sis Time: & 09.05.2019 16:42:13 & & Method. Al120 & \\
\hline & Si [\%] & $\mathrm{Fe}[\%]$ & $\mathrm{Cu}[\%]$ & $\mathrm{Mn}[\%]$ & $\mathrm{Mg}[\%]$ \\
\hline \multirow[t]{2}{*}{$\varnothing$} & 18,32 & 1,054 & 2,093 & 0,184 & 0,072 \\
\hline & $\mathrm{Cr}[\%]$ & Ni [\%] & $\mathrm{Zn}[\%]$ & Ti [\%] & $\mathrm{Pb}[\%]$ \\
\hline \multirow[t]{2}{*}{$\varnothing$} & 0,014 & 0,025 & 0,218 & 0,046 & 0,186 \\
\hline & Sn $[\%]$ & V [\%] & $\operatorname{Sr}[\%]$ & $\mathrm{Zr}[\%]$ & Co [\%] \\
\hline \multirow[t]{2}{*}{$\varnothing$} & 0,026 & 0,011 & $<0,0030$ & 0,0064 & $<0,0030$ \\
\hline & $\mathrm{Ca}[\%]$ & Al [\%] & & & \\
\hline$\varnothing$ & 0,020 & 77,72 & & & \\
\hline
\end{tabular}

Gráfico 2. Resultados de espectrometría de cabezote de referencia.

Fuente: Elaboración propia.

\begin{tabular}{|c|c|c|c|c|c|}
\hline \multicolumn{6}{|c|}{ Bruker Analysis Report } \\
\hline \multicolumn{6}{|c|}{ Sample: } \\
\hline \multicolumn{2}{|c|}{ Analysis Time: } & \multicolumn{2}{|l|}{ 14.11.2019 11:00:40 } & \multicolumn{2}{|l|}{ Method: Al120 } \\
\hline & Si [\%] & $\mathrm{Fe}[\%]$ & $\mathrm{Cu}[\%]$ & Mn [\%] & Mg [\%] \\
\hline \multirow[t]{2}{*}{$\varnothing$} & 19,53 & 1,203 & 1,798 & 0,181 & 0,164 \\
\hline & $\operatorname{Cr}[\%]$ & Ni [\%] & $\mathrm{Zn}[\%]$ & $\mathrm{Ti}[\%]$ & $\mathrm{Pb}[\%]$ \\
\hline \multirow[t]{2}{*}{$\varnothing$} & 0,033 & 0,036 & 0,772 & 0,033 & 0,064 \\
\hline & Sn [\%] & V [\%] & $\mathrm{Sr}[\%]$ & $\mathrm{Zr}[\%]$ & Co $[\%]$ \\
\hline \multirow[t]{2}{*}{$\varnothing$} & 0,018 & 0,020 & $<0,0030$ & 0,0046 & $<0,0030$ \\
\hline & $\mathrm{Ca}[\%]$ & Al [\%] & & & \\
\hline$\varnothing$ & 0,021 & 76,12 & & & \\
\hline
\end{tabular}

Gráfico 3. Resultados de espectrometría de cabezote prototipo.

Fuente: Elaboración propia.

Al momento de la verificación insitu del cabezote prototipo en el motor de combustión interna de dos tiempos de 100cc de una motocicleta Suzuki Ax-100, donde se procedió a encender el motor y se mantuvo a ralentí durante 20 minutos y luego se aceleró a fondo durante 5 minutos y se apagó el motor, se procedió a desmontar el cabezote como se ve en la Figura 7 y se constató que el cabezote soporto las condiciones de funcionamiento siendo estas la temperatura interna de la cámara de combustión al momento de la ignición, así mismo soporto las cargas de compresión como se puede ver en la Figura 8, a continuación (Freire Bravo, 2021).

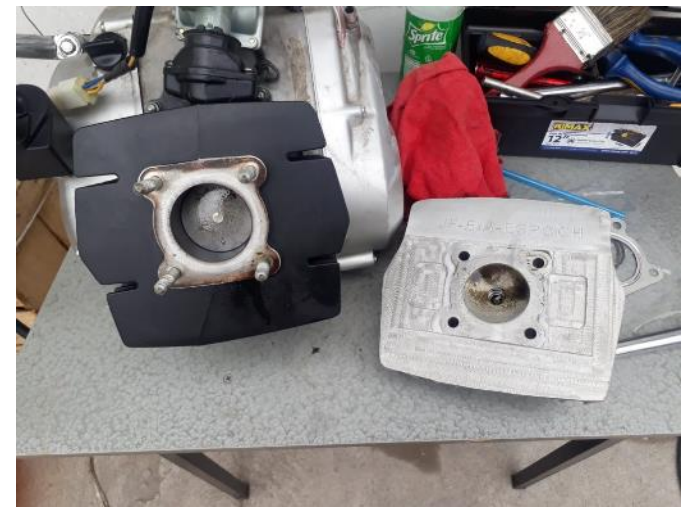

Figura 7. Desmontaje de cabezote puesto a prueba. Fuente: Elaboración propia. 


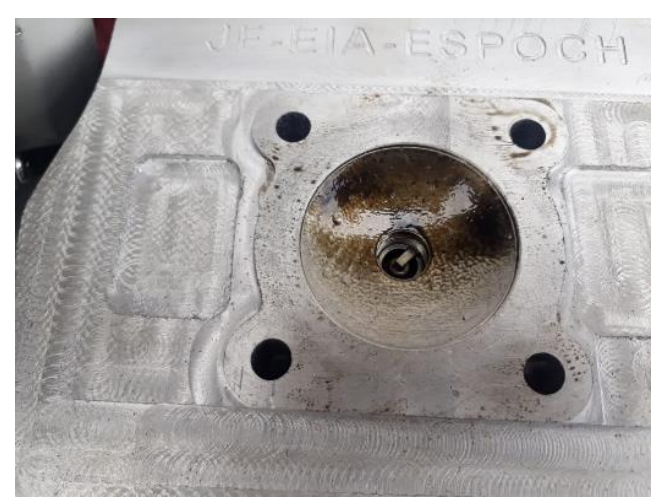

Figura 8. Verificación de anomalías presentadas. Fuente: Elaboración propia.

\section{Conclusiones.}

- Gran parte de los cabezotes para motores de dos tiempos enfriados por aire mono cilindro son de aluminio - silicio en proporciones 70 - 20 respectivamente y pertenecen a la familia de la serie 392 y una dureza que bordea los $108 \mathrm{HB}$.

- El prototipo de cabezote posee 18,32\% de Silicio y 77,72\% de Aluminio, así como también una dureza promedio de $110 \mathrm{HB}$, envejecido naturalmente, considerándose un prototipo funcional y apto para su funcionamiento en campo, ya que cuenta además con las medidas y tolerancias ideales.

- Los ensayos insitu del prototipo de cabezote en un motor de pruebas son positivos ya que soporto todas las cargas térmicas y mecánicas del motor en plena carga.

- La técnica de fundición en verde es un método muy adecuado para obtener partes y piezas automotrices con geometrías complejas ya que la tecina de fundición tradicional es muy limitada.

\section{Referencias bibliográficas.}

Aeade. Asociación de Empresas Automotrices del Ecuador|AUTO Magazine. [en línea]. 2020. Disponible en: https://automagazine.ec/aeade-asociacion-de-empresasautomotrices-del-ecuador/

ASM Handbook, Metallography and microstructures (Asm handbook), ASM Handbook, Volume 9. B-ok.cc [en línea]. 2004. P. 728. Disponible en: https://bok.cc/book/874421/1a8ed2?dsource=recommend

ASTM E10-18, Método de prueba estándar para la dureza Brinell de materiales metálicos, ASTM International, West Conshohocken, PA, 2018, www.astm.org

ASTM E112-13, Métodos de prueba estándar para determinar el tamaño promedio de grano, ASTM International, West Conshohocken, PA, 2013, www.astm.org

ASTM E3-11 (2017), Guía estándar para la preparación de muestras metalográficas, ASTM International, West Conshohocken, PA, 2017, www.astm.org 
ASTM E716-16, Prácticas estándar para el muestreo y la preparación de muestras de aluminio y aleaciones de aluminio para la determinación de la composición química por espectrometría de emisión atómica por chispa, ASTM International, West Conshohocken, PA, 2016, www.astm.org

ASTM, I. ASTM. Annual Book of American Society for Testing and Materials (ASTM) Standards, 4. 2013.

Comunicación, Ú., 2017. Las Normas UNE - Urculo Ingenieros. [en línea] Urculo Ingenieros. Disponible en: http://urculoingenieros.com/las-normasune/\#: :text=Las\%20normas\%20UNE\%20(cuyas\%20siglas,Normalizaci\%C3\%B 3n\%20y\%20Certificaci\%C3\%B3n\%20(AENOR).

Escudero, Secundino. Motores. [en línea]. Madrid: Macmillan Profesional.2011. p. 113; pp. 265-266. Disponible en: https://elibro.net/es/ereader/espoch/101844?page=265

García, J. Estudio de la influencia de diferentes diseños de los sistemas de alimentación y compensación en la fundición en arena. [en línea]. 2016. Disponible en: http://bibing.us.es/proyectos/abreproy/60372/fichero/PFC+Portada+e+\%C3\%AD ndice.pdf

Gmbh, Pilz, Normas internacionales y nacionales (IEC, ISO, DIN, ...). Pilz MX. Pilz.com [en línea]. 2018. Disponible en: https://www.pilz.com/es-MX/knowhow/lawstandards-norms/standards/iso-iec-standards

Millán Delgado, Federico, Fabricación y caracterización de la aleación de aluminio reciclado con adición de silicio particulado. Core.ac.uk [en línea]. 2016. p. 21. Disponible en: https://core.ac.uk/display/77278639

Monroy, M. E., Arciniegas, J. L., \& Rodríguez, J. C. Propuesta Metodológica para Caracterizar y Seleccionar Métodos de Ingeniería Inversa. (2013). Información Tecnológica, 24(5), 23-30. https://doi.org/10.4067/S0718-07642013000500004

Rocabruna, J. Manufactura, Ingeniería Y Tecnología, 5Ta Edición - S. Kalpakjian, S. R. Schmid. [en línea]. 2018. Academia.edu. Disponible en: https://www.academia.edu/39049497/Manufactura_Ingenier\%C3\%ADa_y_Tecno $\log \%$ C3\%ADa_5ta_Edici\%C3\%B3n_S_Kalpakjian_S_R_Schmid

Villarreal Bolaños, Carlos Alfonso, Alternativa para la construcción de autopartes vehicular por medio de la ingeniería inversa e impresión 3D. Caso de estudio tapa de distribución inferior del Chevrolet Spark 2015. [en línea]. Universidad internacional SEK. 2019. pp. 22-24. Disponible en: http://repositorio.uisek.edu.ec/handle/123456789/3303 
Wang, R., \& Lu, W. Direct Electrolytic Al-Si Alloys (DEASA)-An Undercooled Alloy Self-Modified Structure and Mechanical Properties. (2012). Realizado por: Janis Kleperis and Vladimir Linkov, 107.

Bravo Morocho, V. D., Abarca Pérez, E. P., \& Miguel Angel, E. G. (2020). Obtención de las propiedades mecánicas de un cabezote, biela y pistón de motor mono-cilindrico de dos tiempos 100cc, aplicando ingeniería inversa. ConcienciaDigital, 3(3), 510527. Disponible en: https://doi.org/10.33262/concienciadigital.v3i3.1352.

Freire Bravo, Jhon Jesus. Manufactura de un cabezote de un prototipo de motor de dos tiempos mono cilíndrico 100cc aplicando ingeniería inversa y el método CAD/CAM. Escuela Superior Politécnica De Chimborazo ESPOCH. 2021.

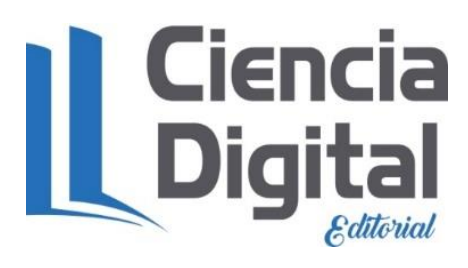




\section{PARA CITAR EL ARTÍCULO INDEXADO.}

Bravo Morocho, V. D., Abarca Pérez, E. P., López Ortíz, S. A., \& Freire Bravo, J. J. (2021). Manufactura de un prototipo cabezote de motor de dos tiempos mono cilíndrico $100 \mathrm{cc}$ aplicando ingeniería inversa y el método CAD/CAM. ConcienciaDigital, 4(3.1), 190-206. https://doi.org/10.33262/concienciadigital.v4i3.1.1823

\section{LCiencia}

El artículo que se publica es de exclusiva responsabilidad de los autores y no necesariamente reflejan el pensamiento de la Revista Ciencia Digital.

El artículo queda en propiedad de la revista y, por tanto, su publicación parcial y/o total en otro medio tiene que ser autorizado por el director de la Revista Ciencia Digital.
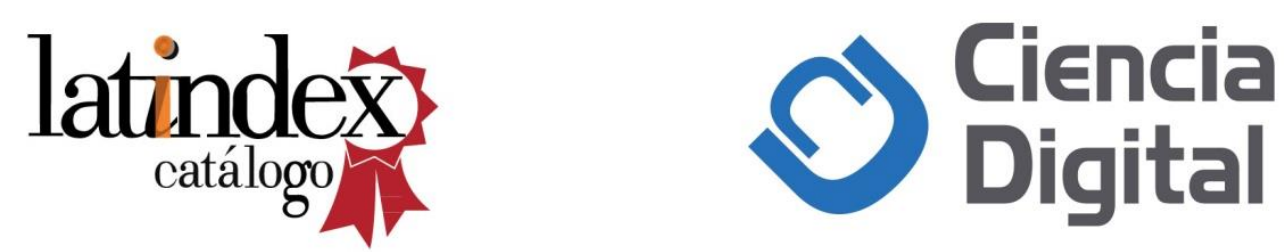